\title{
Improvement of sagittal parameters in patients with cervical spondylotic myelopathy is associated with outcome after anterior cervical discectomy and fusion
}

\section{Yefu Xu ( $\nabla$ xuyefu01@163.com )}

Southeast University Zhongda Hospital https://orcid.org/0000-0003-0794-2116

\section{Sangni Liu}

Southeast University Zhongda Hospital

\section{Feng Wang}

Southeast University Zhongda Hospital Department of Spine Surgery

\section{Xiaotao Wu}

Southeast University Zhongda Hospital Department of Spine Surgery

\section{Research article}

Keywords: Anterior cervical decompression and fusion (ACDF), Cervical lordosis (CL), Thoracic 1 slope (T1S), sagittal vertical axial (SVA), NDI (neck disability index) score

Posted Date: January 15th, 2020

DOl: https://doi.org/10.21203/rs.2.20883/v1

License: (c) (i) This work is licensed under a Creative Commons Attribution 4.0 International License. Read Full License 
Title: Improvement of sagittal parameters in patients with cervical spondylotic myelopathy is associated with outcome after anterior cervical discectomy and fusion;

Authors: 1. Yefu $\mathrm{Xu}^{1,2} ;$ 2. Sangni Liu ${ }^{1,2} ; 3$. Feng Wang ${ }^{1,2}$; 4. Xiaotao $\mathrm{Wu}^{1,2}$;

${ }^{1}$ Zhongda Hospital of Southeast University Department of Spine Surgery, ${ }^{2}$ Southeast University

Corresponding author identity: Yefu Xu, Resident of Zhongda Hospital; Xiaotao Wu, Chief physician of Zhongda Hospital;

Author affiliation: Zhongda Hospital of Southeast University Department of Spine Surgery, Nanjing 210009, China;

Addresses: No.87 Dingjiaqiao, Gulou district, Nanjing 210009, China

E-mail: xuyefu01@163.com;

Telephone number: $86+15150687353$;

ORCID codes: 0000-0003-0794-2116 


\section{Abstract:}

Background : After ACDF, cervical lordosis loss, high C2-7 SVA and postoperative T1S will worsen the surgical prognosis.

Therefore, this study evaluated the changes of cervical parameters after surgery and correlation with surgical prognosis.

Methods : This study enrolled 212 cervical compressive myelopathy patients who underwent ACDF. We collect gender, age, BMI, NDI scores and cervical parameters. And then, we compare the changes of parameters after surgery, and explore the correlation between parameters and NDI score.

Results : C2-7 Cobb Angle, SVA, T1S and TS-CL were all improved after surgery, but T1S at the last follow-up was larger than that before the surgery. T1S is positively correlated with TIA ( $\mathrm{p}=0.018, \mathrm{r}=0.081), \mathrm{CL}(\mathrm{p}<0.01, \mathrm{r}=0.150)$ and SVA $(\mathrm{p}<0.01, \mathrm{r}=0.131)$, but negatively correlated with SCA $(\mathrm{p}<0.01, \mathrm{r}=-0.166)$. We also found that TIA was highly correlated with the sum of T1S $+\mathrm{NT}$ ( $<<0.01$, $\mathrm{r}=0.459)$. Preoperative NDI was negatively correlated with preoperative c2-7 Cobb Angle $(\mathrm{r}=-0.147, \mathrm{p}=0.033)$, positively correlated with SVA ( $\mathrm{r}=0.157, \mathrm{p}=0.022)$. The last follow-up NDI score was negatively correlated with $\mathrm{C} 2-7$ Cobb Angle $(\mathrm{r}=-0.222 \mathrm{p}=0.001)$, positively correlated with SVA $(r=0.498, \mathrm{p}<0.001)$ and TS-CL $(\mathrm{r}=0.165, \mathrm{p}=0.016)$. The risk factors with poor follow-up NDI score (the last follow-up NDI score greater than $30 \%$ ) were SVA $(B=0.337, \mathrm{P}=<0.001), \mathrm{CL}(\mathrm{B}=-0.147, \mathrm{P}=0.029)$ and gender $(\mathrm{B}=2.153$, $\mathrm{P}=0.017)$.

Conclusions : After ACDF, most postoperative cervical parameters have improved, but T1S deteriorated over time. There was a close correlation between cervical sagittal parameters. Preoperative NDI score was correlated with c2-7 Cobb Angle and SVA. Follow-up NDI score was correlated with CL, TS-CL and SVA. High SVA, loss of cervical lordosis and male patient were risk factors for the deterioration of postoperative NDI score.

Keywords : Anterior cervical decompression and fusion (ACDF); Cervical lordosis (CL), Thoracic 1 slope (T1S), sagittal vertical axial (SVA) ; NDI (neck disability index) score;

\section{Background}

The sagittal balance of the physiologically upright spine maintains intervertebral alignment with minimal energy expenditure ${ }^{1}$. The primary task of the cervical spine is to maintain horizontal gaze ${ }^{2}$. Cervical imbalance may interfere with sagittal alignment and induce compensatory mechanisms, leading to increased energy expenditure, muscle fatigue and neck pain ${ }^{3}$. In addition, excessive loading of cervical endplate accelerate cervical degeneration, leading to cervical deformity and spinal cord compression. ACDF can decompress the anterior 
spinal cord, reduce surgical bleeding, and maintain spinal stability ${ }^{4}$. As the gold standard for the treatment of CSM, it has significant long-term clinical effects. Studies have shown that cervical sagittal parameters after ACDF have been significantly improved, but some studies observe that some parameters of postoperative follow-up have deteriorated.

ACDF can relieve symptoms through decompression and release spinal cord or nerve root. However, postoperative symptoms such as loss of cervical angle, excessive C2-C7 SVA, and more than $30^{\circ} \mathrm{T} 1 \mathrm{~S}$ may worsen the postoperative health-related quality of life (HRQOL) ${ }^{5}$. Therefore, cervical sagittal balance has been taken as one of the reference bases for the preoperative planning. However, there is no clear conclusion about the changes of sagittal parameters before and after ACDF in patients with CSM and the relationship between cervical parameters and surgical prognosis. Therefore, this study aims to compare the changes of parameters after surgery, and analyze the correlation between cervical parameters and patient NDI scores.

\section{Methods}

\subsection{Subjects}

(1) Inclusion criteria: the preoperative diagnosis of cervical spondylotic myelopathy was clear. There should be at least one symptom or sign of CSM, and the radiological changes should be consistent with clinical diagnosis. The patients should underwent ACDF in our hospital, with complete data and clear anatomical markers in X-ray image. (2) Exclusion criteria: previous history of cervical surgery, clear history of trauma, spinal tumor, infection, ankylosing spondylitis, congenital deformity, incomplete clinical and imaging data, incomplete X-ray projection of the neck, no imaging data of postoperative follow-up.

A retrospective study was conducted on patients who received surgery for CSM in our hospital from May 2014 to June 2018. A total of 212 patients met the above criteria and were included in this study. There were 147 male cases with an average age of $53.78 \pm 11.47$ years, and 65 female patients with an average age of $52.27 \pm 9.20$ years. Data were collected including gender, age, symptom duration, surgical method, cervical parameters, etc.

\subsection{Measures}

\subsubsection{Evaluation of radiological findings}

Lateral cervical X-ray examination: the patients maintain horizontal gaze with the neck in the center. Measurement parameters include C2-7 Cobb Angle (Cervical lordosis, CL), Thoracic 1 slope (T1S), C2-7 sagittal vertical axial (SVA), thoracic inlet Angle (TIA), neck tilt (NT), spine-cranial Angle (SCA), and T1S-CL ( as figure 1 and figure 2). The measurement methods are shown in table 1 . The above parameters were measured twice 
independently by two spine orthopedic surgeons, and the average values of the two measurements were taken for further analysis. The interobserver reliabilities analyzed by the interclass correlation coefficient (ICC) $(2,1)$ showed close correlations of more than 0.8 for each radiological parameter $(\mathrm{r}=0.827)$. Imaging data collection was divided into four stages: preoperative ( 1 to 5 days before surgery in the hospital), postoperative ( 1 to 3 days after surgery in the hospital), Follow-up of 3 months ( 3 months after surgery during outpatient), and the last follow-up (12 months or more after surgery during outpatient).

\subsubsection{Patient characteristics measures}

Fusion segments, internal fixation materials (titanium cage or not), BMI (body mass index), gender, age, diabetes history, symptom duration, smoking and drinking history were collected through the hospital record system. NDI scores were collected at preoperative and the last follow-up. The patient voluntarily filled in the cervical NDI scale, and the total score was calculated by researchers. The NDI score was used to evaluate the the severity of CSM.

\section{3 statistical analysis}

SPSS 21.0 (SPSS, Chicago) statistical software was used for statistical analysis of the measurement results. Descriptive analysis of parameters preoperative, postoperative and follow-up was conducted, and One-way ANOVA was used for comparison. Independent sample t-test was used to compare preoperative and follow-up NDI scores. Spearman analysis was used for inter-parameter correlation analysis. In addition, Spearman analysis was also used for correlation analysis between NDI scores and each parameter. P value less than 0.05 has a statistically significant difference. Logistic analysis was conducted for all data and postoperative NDI score. Whether the NDI score was greater than 30 in the last follow-up was taken as the assessment result. The last follow-up cervical parameters and patient characteristics data were analyzed by binary logistic.

\section{Results}

\section{Patient demographic and radiological parameters}

Finally, 212 patients were followed up (mean follow-up time: 17.6 months), including 118 males (mean age: $53.78 \pm 11.47$ years) and 94 female patients (mean age: $52.27 \pm 9.20$ ). The average BMI were $23.85 \pm 2.65$, and average symptom duration were $10.77 \pm 17.61$ months. There were 10 cases of c3-4 segment fusion, 16 cases of c4-5 segment fusion, 44 cases of c5-6 segment fusion, 6 cases of c6-7 segment fusion, 4 cases of c3-5 segment fusion, 40 cases of c4-6 segment fusion, 48 cases of c5-7 segment fusion, 18 cases of c3-6 segment fusion, 22 cases of c4-7 segment fusion, and 4 cases of c3-7 segment fusion. There were 31 patients with smoking, 11 patients with alcoholism and 23 diabetic patients. 


\section{Comparison of Preoperative parameters and postoperative parameters}

The value of each stage parameters and the comparison of various parameters such as table 2. Immediately postoperative and follow-up C2 - 7 Cobb Angle increases every period compared with preoperative. Immediately postoperative T1S was less than that before surgery, but the last follow-up T1S was larger than preoperative. Immediately postoperative and 3 months follow-up SVA reduced compared with preoperative. Immediately postoperative and follow-up TS-CL reduced every period compared with preoperative (as figure 3). There was no significant difference between 3 months follow-up T1S and preoperative. Similarly, there was no significant difference between the last follow-up SVA and preoperative. Preoperative SCA, NT, TIA has no obvious difference with postoperative (as figure 4).

\section{Parameters correlation}

The correlation among parameters is shown in table 3 , T1S is positively correlated with TIA $(\mathrm{p}=0.018$, $\mathrm{r}=0.081), \mathrm{CL}(\mathrm{p}<0.01, \mathrm{r}=0.150)$ and SVA $(\mathrm{p}<0.01, \mathrm{r}=0.131)$, T1S is negatively correlated with $\mathrm{SCA}(\mathrm{p}<0.01$, $\mathrm{r}=-0.166)$, In addition, SCA is negatively correlated with CL ( $\mathrm{p}<0.01, \mathrm{r}=-0.502)$, SVA is positively correlated with TS-CL $(p<0.01, r=0.160)$, and negatively correlated with CL $(p=0.012, r=-0.086)$. We also found that TIA was highly correlated with the sum of T1S+NT $(\mathrm{p}<0.01, \mathrm{r}=0.459)$.

\section{Correlation between parameters and NDI scores}

Preoperative NDI scores was $21.91 \pm 13.96$, the last follow-up NDI scores was $15.73 \pm 11.12$. The last follow-up NDI scores was reduced than preoperative $(\mathrm{p}<0.01)$. The relationship between NDI score and parameters is shown in table 4. Preoperative NDI was negatively correlated with preoperative c2-7 Cobb Angle $(\mathrm{r}=-0.147, \mathrm{p}=0.033)$, positively correlated with SVA $(\mathrm{r}=0.157, \mathrm{p}=0.022)$. It was not significantly correlated with preoperative T1S, TS-CL, SCA, NT, and TIA. The last follow-up NDI score was negatively correlated with follow-up C2-7 Cobb Angle ( $r=-0.222 \mathrm{p}=0.001)$ (as figure 5), positively correlated with SVA $(\mathrm{r}=0.498, \mathrm{p}<0.001$ ) (as figure 6) and TS-CL ( $\mathrm{r}=0.165, \mathrm{p}=0.016$ ) (as figure 7). The last follow-up NDI score was not significantly correlated with follow-up T1S, SCA, NT, and TIA. The risk factors with poor follow-up NDI score (the last follow-up NDI score greater than 30\%) were analyzed by binary logistic analysis (as table 5), and the results showed that $\mathrm{SVA}(\mathrm{B}=0.337, \mathrm{P}=<0.001), \mathrm{CL}(\mathrm{B}=-0.147, \mathrm{P}=0.029)$ and gender $(\mathrm{B}=2.153, \mathrm{P}=0.017)$ were the risk factors affecting NDI score greater than $30 \%$.

\section{Discussion}

In order to maintain horizontal gaze of the head, degenerative cervical appears compensatory changes, and the sagittal parameters of cervical-thoracic segment will also change correspondingly. In asymptomatic 
individuals, there was also a strong association between cervical parameters. Lee ${ }^{6}$ measured TIA,T1S and NT of 77 asymptomatic volunteers at $69.5^{\circ}, 25.7^{\circ}$ and $43.7^{\circ}$, respectively, there was a significant correlation between TIA and T1S $(r=0.694)$. There was a significant correlation between the thoracic inlet pathway and cranial-cervical sagittal balance. In order to maintain physiological NT at about $44^{\circ}$,TIA and T1S increased correspondingly, The relationship among three parameters was approximately as follows: TIA=T1S+NT. In our study, it was found that there was a significant correlation between TIA and T1S+NT in patients with CSM $(\mathrm{r}=0.459, \mathrm{P}<0.001)$. This relationship is similar to that pelvic incidence is the sum of sacral slope and pelvis tilt.

It was found that among the measured parameters of cervical-thoracic segment, someone was significantly correlated with other parameters. Therefore, these parameters could be taken as the preferred criteria for measurement. Some studies believed that T1S was the key factor determining cervical sagittal balance, and was closely related to other parameters. Le $\mathrm{Huec}^{7}$ found a significant correlation between T1S and SCA in asymptomatic volunteers $(\mathrm{r}=-0.818, \mathrm{P}<0.001)$. $\mathrm{Staub}^{8}$ suggested that $\mathrm{T} 1 \mathrm{~S}$ was significantly correlated with $\mathrm{C} 2-7$ lordosis $(\mathrm{r}=0.815)$. Ames et $\mathrm{al}^{9}$ found a significant correlation between T1S and C2-7 SVA $(\mathrm{r}=0.44, \mathrm{p}<0.001)$. Similarly, our study found that T1S was significantly correlated with SCA, TIA, CL and SVA. As a bridge between the cervical and thoracic segments, T1S was very sensitive to changes in cervical spine sequences, which could be used as an important indicator for evaluating cervical balance in the future.

Anterior cervical discectomy and fusion (ACDF) is one of the most common spinal surgeries ${ }^{10}$, which can be used to correct cervical kyphosis and improve the symptoms of nerve root and spinal cord compression. Changes in cervical alignment before and after surgery have a direct impact on health-related quality of life (HRQOL) ${ }^{11}$. By analyzing the correlation between NDI score and cervical parameters at the corresponding period, it was found that C2-7 lordosis, SVA, TS-CL and NDI score were significantly correlated. Binary logistic analysis suggested that gender, CL and SVA were risk factors for postoperative NDI deterioration. In addition, we also found that the last follow-up T1S was bigger than postoperative, this could mean that some cervical parameters will be deteriorated over time, and sagittal balance will be affected. Though the last follow-up NDI score compared with preoperative obviously improved, but the long-term prognosis for influence still exists, may increase the risk of adjacent segment disease.

Prior to this, studies have found that ${ }^{12}$ when $\mathrm{T} 1 \mathrm{~S}>40^{\circ}$, immediately after ACDF, SVA improved, but deteriorated 2 years later. Huang ${ }^{13}$ conducted 2-year follow-up on 42 patients after cervical spine surgery, and found that preoperative T1S was correlated with preoperative NDI score $(r=0.413)$, and postoperative T1S was correlated with follow-up NDI score $(\mathrm{r}=0.534)$. It can be considered that sagittal parameters after ACDF are 
related to clinical prognosis, and too large T1S is a risk factor affecting surgical prognosis. T1S can be used as a reference value to judge the sagittal balance of cervical. Hyun ${ }^{14}$ conducted a follow-up of at least 5 years for patients after ACDF, and found that C2-7 SVA and TS-CL were positively correlated with NDI score, and TS-CL was positively correlated with C2-7 SVA. Regression analysis showed that C2-7 SVA value of $40 \mathrm{~mm}$ and $70 \mathrm{~mm}$ corresponds to TS-CL value of $20^{\circ}$ and $25^{\circ}$. In addition, $\mathrm{LAN}^{15}$ found that changes in postoperative cervical parameters were related to HRQOL through measurement of TS-CL and C2-7 SVA of patients with 2 adjacent segments ACDF. He believed that large TS-CL was related to cervical dislocation, and TS-CL might be a more important predictor of cervical deformity than C2-7 SVA. Although our study found that TS-CL and SVA were correlated with NDI score, the correlation between T1S and NDI score was not confirmed.

Kwon ${ }^{16}$ found that patients with cervical kyphosis after ACDF had more obvious neck pain symptoms. Similarly, this study found that cervical kyphosis was negatively correlated with NDI score, and excessive cervical kyphosis would cause neck discomfort. Kim et al ${ }^{17}$ conducted 1-year follow-up of postoperative patients and found that changes of C2-7 lordosis and SVA were significantly different from those before surgery, which were related to improvement of cervical spine sequence. We found a certain correlation between cervical lordosis and NDI score, and identified cervical lordosis loss as one of the risk factors for NDI deterioration in binary logistic analysis. However, Villavicencio et al ${ }^{18}$ found no significant correlation between changes in cervical kyphosis and clinical outcomes in a randomized controlled trial of patients after ACDF surgery.

Tang ${ }^{19}$ suggested that increased SVA was associated with low HRQOL score. SVA greater than 40mm was associated with poor NDI score, and affected postoperative recovery of cervical. Therefore, cervical sagittal deviation should not be too large during cervical vertebra orthopedics. Tamai ${ }^{20}$ found that in patients with cervical imbalance, C7-T11 lordosis angle was small and T1S increased. The muscles of C7 segments would show degenerative changes, and some patients had cervical spondylolisthesis. They believed that advanced age, male patient and cSVA $\geq 40 \mathrm{~mm}$ were important factors causing cervical imbalance. The above arguments are highly consistent with the logistic regression results of our study. We found that male patients and high SVA were risk factors for poor postoperative prognosis. Furthermore, cervical lordosis loss was also found to be one of the risk factors.

This study for cervical parameters changes and correlation to describe the results of complete. But because of postoperative NDI score collection difficulty, some patients were removed due to loss of follow-up, resulting in errors in the analysis of the correlation between NDI score and cervical parameters. In addition, cervical parameters measures need to be further improved. Some samples were excluded and a large amount of data was 
lost due to the nonstandard posture during the lateral cervical spine radiographs or the postoperative inability to stand. In this case, the patients who met the inclusion criteria were in better physical condition, so the NDI score may be relatively small compared with the actual results, which should be avoided as far as possible in the design of future clinical studies.

The primary task for the spine a whole, and not only for maintain horizontal gaze. When the lumbar and or thoracic spine change during life time, the cervical spine has to adapt in order to keep this gaze. As such, a normal cervical lordosis does not exist (appears in about $50 \%$ of asymptomatic individuals). Therefore, it makes no sense to measure a lot of cervical parameters, without measuring the global balance of the spine. Therefore, in future patients after ACDF, full-length radiography of spine, as well as cervical and thoracolumbar parameters, should be measured for analysis. For sure, it seems probable that while fixing the spine, we should take into account the type of spine, the global balance and the compensation mechanisms. Finally, these cervical parameters should be affected by the spinopelvic alignment. In patients with CSM, loss of physical activity might result in muscle weakness, which might induce spinal imbalance. Outcome measure of CSM need be added in future study.

\title{
Conclusion
}

After ACDF, most postoperative cervical parameters have improved, but T1S deteriorated over time. There was a close correlation between cervical sagittal parameters. Preoperative NDI score was correlated with c2-7 Cobb Angle and SVA. Follow-up NDI score was correlated with CL, TS-CL and SVA. High SVA, loss of cervical lordosis and male patient were risk factors for the deterioration of postoperative NDI score.

\begin{abstract}
Abbreviations:
Anterior cervical decompression and fusion (ACDF); cervical spondylotic myelopathy(CSM); Cervical lordosis (CL), Thoracic 1 slope (T1S), sagittal vertical axial (SVA); Thoracic 1 slope minus Cervical lordosis (TS-CL); Spine-cranial angle (SCA); Thoracic inlet angle (TIA); Neck tilt (NT); NDI (neck disability index) score; health-related quality of life (HRQOL);
\end{abstract}

\section{Declarations}

\section{Ethics approval and consent to participate}

The study protocol and data use were approved by Clinical research ethics committee of Zhongda Hospital, Affiliated to Southeast University. Project code: 2019ZDSYLL175-P01

\section{Consent for Publication}

Not Applicable.

\section{Availability of data and material}


All relevant data are within the manuscript, tables and its supplementary files.

\section{Competing interests}

The authors declare that they have no competing interests.

\section{Funding}

Funding received came from National Natural Science Foundation of China (Project approval number: 81871810). The funders had no role in study design, data collection and analysis, decision to publish, and preparation of the manuscript.

\section{Authors' contributions}

YFX, FW and XTW developed the study design. YFX and SNL collected data and measured parameters. Follow-up data were collected by YFX and SNL. YFX and FW performed the data analysis and statistical evaluation. YFX drafted the manuscript. All authors read and approved the manuscript.

\section{Acknowledgements}

Not Applicable. 


\section{References}

1 Vital J M, Senegas J (1986) Anatomical bases of the study of the constraints to which the cervical spine is subject in the sagittal plane. A study of the center of gravity of the head.[J] .Surg Radiol Anat 8: 169-73. https://doi.org/10.1007/bf02427845

2 Diebo B G, Challier V, Henry J K , et al (2016) Predicting Cervical Alignment Required to Maintain Horizontal Gaze Based on Global Spinal Alignment[J]. Spine 41(23):1. https://doi.org/10.1097/BRS.0000000000001698

${ }^{3}$ Dudli S, Fields A J , Samartzis D , et al (2016) Pathobiology of Modic changes[J]. European Spine Journal 25(11):3723-3734. https://doi.org/10.1007/s00586-016-4459-7

4 Ames C P , Smith J S , Scheer J K, et al (2012) Impact of spinopelvic alignment on decision making in deformity surgery in adults[J]. Journal of Neurosurgery: Spine 16(6):547-564. https://doi.org/10.3171/2012.2.SPINE11320

${ }^{5}$ Youn M S , Shin J K, Goh T S , et al (2016) Relationship between cervical sagittal alignment and health-related quality of life in adolescent idiopathic scoliosis[J]. European Spine Journal 25(10):3114-3119. https://doi.org/10.1007/s00586-016-4488-2

${ }^{6}$ Lee S H , Kim K T , Seo E M, et al (2012) The Influence of Thoracic Inlet Alignment on the Cranio-cervical Sagittal Balance in Asymptomatic Adults [J]. Journal of Spinal Disorders \& Techniques 25(2):E41-E47. https://doi.org/10.1097/BSD.0b013e3182396301

7 Le Huec J C, Demezon H, Aunoble S (2015) Sagittal parameters of global cervical balance using EOS imaging: normative values from a prospective cohort of asymptomatic volunteers [J]. European Spine Journal 24(1):63-71. https://doi.org/10.1007/s00586-014-3632-0

8 Blake N. Staub, Renaud Lafage, Han Jo Kim, et al (2018) Cervical mismatch: the normative value of T1 slope minus cervical lordosis and its ability to predict ideal cervical lordosis [J]. Journal of Neurosurgery: Spine 2018 10 05;30(1). https://doi.org/10.3171/2018.5.SPINE171232

9 Ames C P , Blondel B , Scheer J K , et al (2013) Cervical Radiographic Alignment: comprehensive assessment techniques and potential importance in cervical myelopathy[J]. Spine 38:S149-S160. https://doi.org/10.1097/BRS.0b013e3182a7f449

10 Jiang L, Tan M , Dong L, et al (2015) Comparison of Anterior Decompression and Fusion With Posterior Laminoplasty for Multilevel Cervical Compressive Myelopathy: A Systematic Review and Meta-Analysis[J]. Clinical Spine Surgery 28(8):282. https://doi.org/10.1097/BSD.0000000000000317

11 Protopsaltis T S, Scheer J K, Terran J S, et al (2013) How the neck affects the back: changes in regional cervical sagittal alignment correlate to HRQOL improvement in adult thoracolumbar deformity patients at 2-year follow-up [J]. Journal of Neurosurgery Spine 13(9):S97-S98. https://doi.org/10.3171/2014.11.SPINE1441

12 Oe S , Yamato Y , Togawa D , et al (2016) Preoperative T1 Slope More Than $40^{\circ}$ as a Risk Factor of Correction Loss in Patients With Adult Spinal Deformity[J]. Spine 41(19):E1168. https://doi.org/10.1097/BRS.0000000000001578

${ }^{13}$ Huang Y, Lan Z, Xu W (2019) Analysis of sagittal alignment parameters following anterior cervical hybrid decompression and fusion of multilevel cervical Spondylotic myelopathy [J]. BMC Musculoskeletal Disorders 20(1). https://doi.org/10.1186/s12891-018-2378-y

${ }_{14}$ Hyun S J, Han S , Kim K J (2018) Assessment of T1 Slope Minus Cervical Lordosis and C2-7 Sagittal Vertical Axis Criteria of a Cervical Spine Deformity Classification System Using Long-Term Follow-up Data After Multilevel Posterior Cervical Fusion Surgery.[J]. Operative Neurosurgery. https://doi.org/10.1093/ons/opy055

15 Lan Z, Huang Y, Xu W (2019) Relationship Between T1 Slope Minus C2-7 Lordosis and Cervical Alignment Parameters After Adjacent 2-Level Anterior Cervical Diskectomy and Fusion of Lower Cervical Spine. [J]. World Neurosurgery. https://doi.org/10.1016/j.wneu.2018.11.016

${ }^{16}$ Kwon B , Kim D H , Marvin A, et al (2005) Outcomes following anterior cervical discectomy and fusion: the role of interbody disc height, angulation, and spinous process distance[J]. Journal of Spinal Disorders \& Techniques 18(4):304-308. https://doi.org/10.1097/01.bsd.0000167359.10683.b1

17 Kim, H. J.Choi, B. W.Park, J.Pesenti, S.Lafage, V (2019) Anterior cervical discectomy and fusion can restore cervical sagittal alignment in degenerative cervical disease [J]. Eur J Orthop Surg Traumatol. https://doi.org/10.1007/s00590-019-02386-7

18 Villavicencio A T , Babuska J M, Ashton A, et al (2011) Prospective, Randomized, Double-Blind Clinical Study Evaluating the Correlation of Clinical Outcomes and Cervical Sagittal Alignment [J]. Neurosurgery 68(5):1309-1316. https://doi.org/10.1227/NEU.0b013e31820b51f3

19 Tang Jessica A,Scheer Justin K,Smith Justin S et al (2015) The impact of standing regional cervical sagittal alignment on outcomes in posterior cervical fusion surgery. [J] Neurosurgery. https://doi.org/10.1227/01.neu.0000462074.66077.2b

${ }^{20}$ Tamai Koji,Romanu Joshua,Grisdela Phillip et al (2018) Small C7-T1 lordotic angle and muscle degeneration at C7 level were independent radiological characteristics of patients with cervical imbalance: a propensity score-matched analysis.[J] .Spine J 18: 1505-1512. https://doi.org/10.1016/j.spinee.2018.01.012 


\section{Figures}

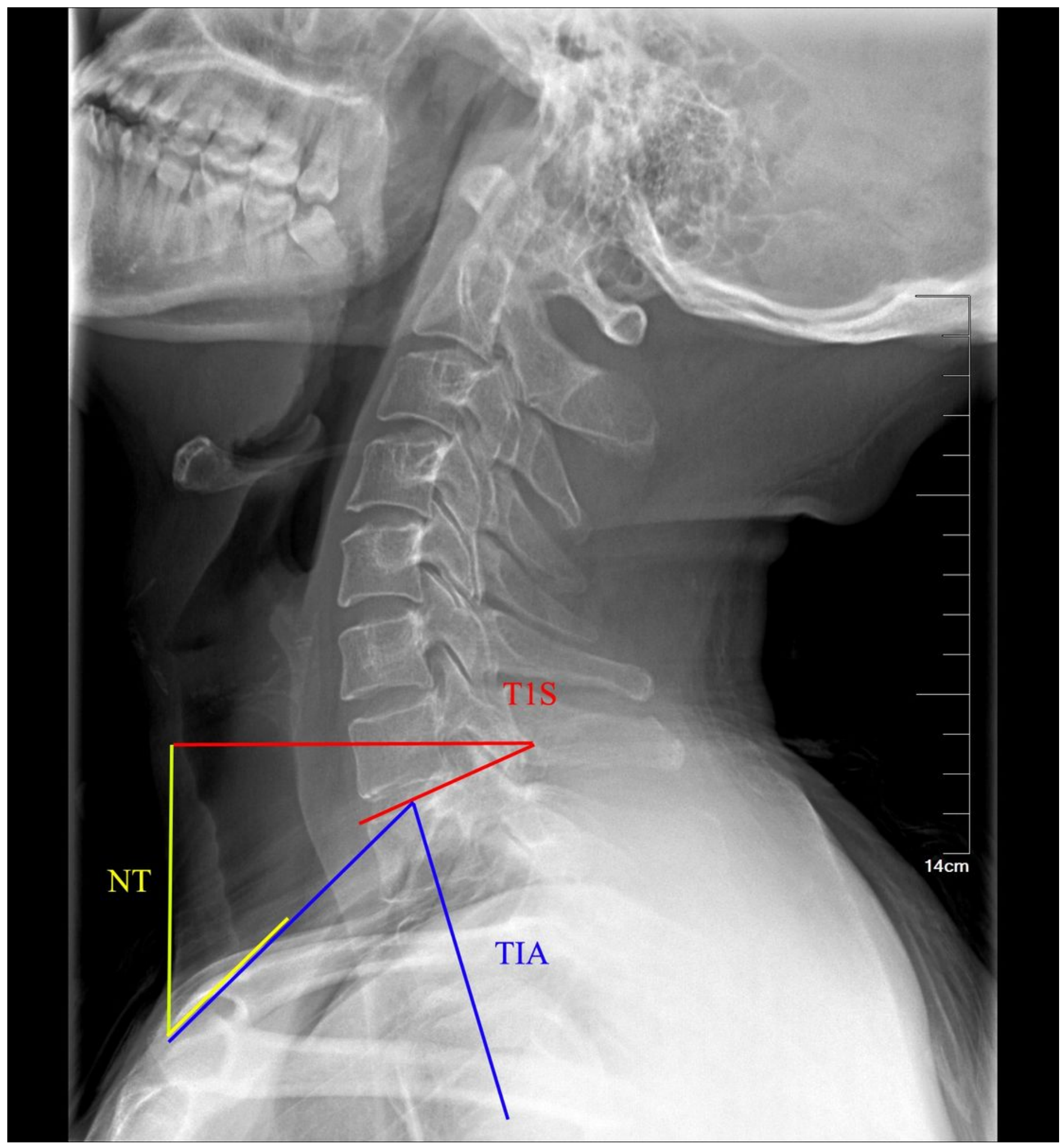

\section{Figure 1}

Method for measuring T1S, NT and TIA. T1S: Angle between a horizontal line and the superior endplate of T1; NT: Angle formed by the reference vertical line drawn in the upper end of the sternum and a line connecting the center of the T1 upper end plate and the upper end of the sternum; TIA: Angle formed by a 
line perpendicular to the superior endplate of $\mathrm{T} 1$ and a line connecting the center of the $\mathrm{T} 1$ upper end plate and the upper end of the sternum.

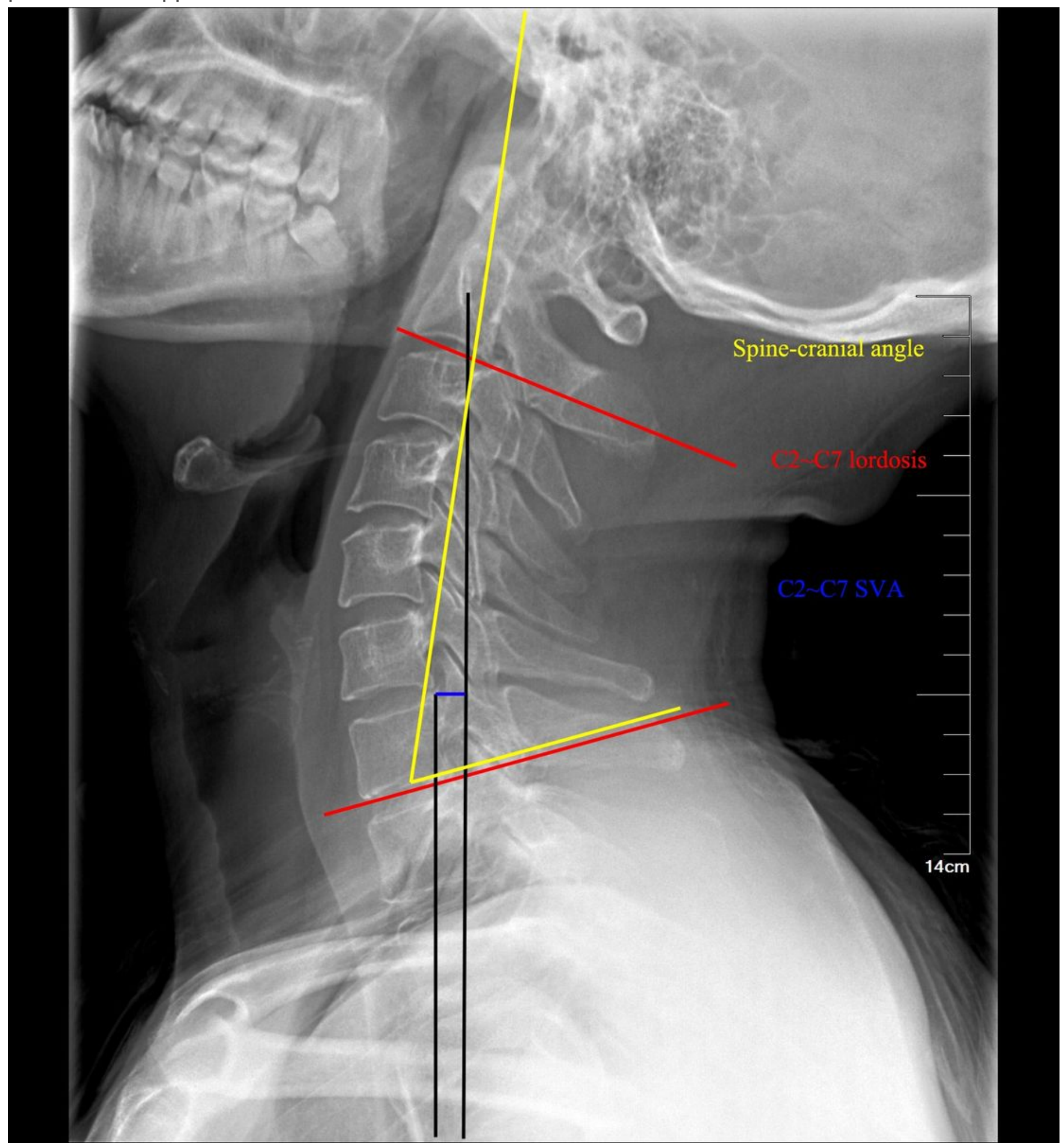

Figure 2

Method for measuring C2-C7 lordosis, C2-C7 SVA and Spine-cranial angle. C2-C7 lordosis: Angle between the lower plate of $\mathrm{C} 2$ and the lower plate of $\mathrm{C7} ; \mathrm{C} 2-\mathrm{C} 7 \mathrm{SVA}$ : The distance from the posterior, superior corner of $\mathrm{C} 7$ to the plumb-line from the centroid of C2; Spine-cranial angle :The angle is defined between 
the $\mathrm{C} 7$ slope and the straight line joining the middle of the $\mathrm{C} 7$ end plate and the middle of the sella turcica.

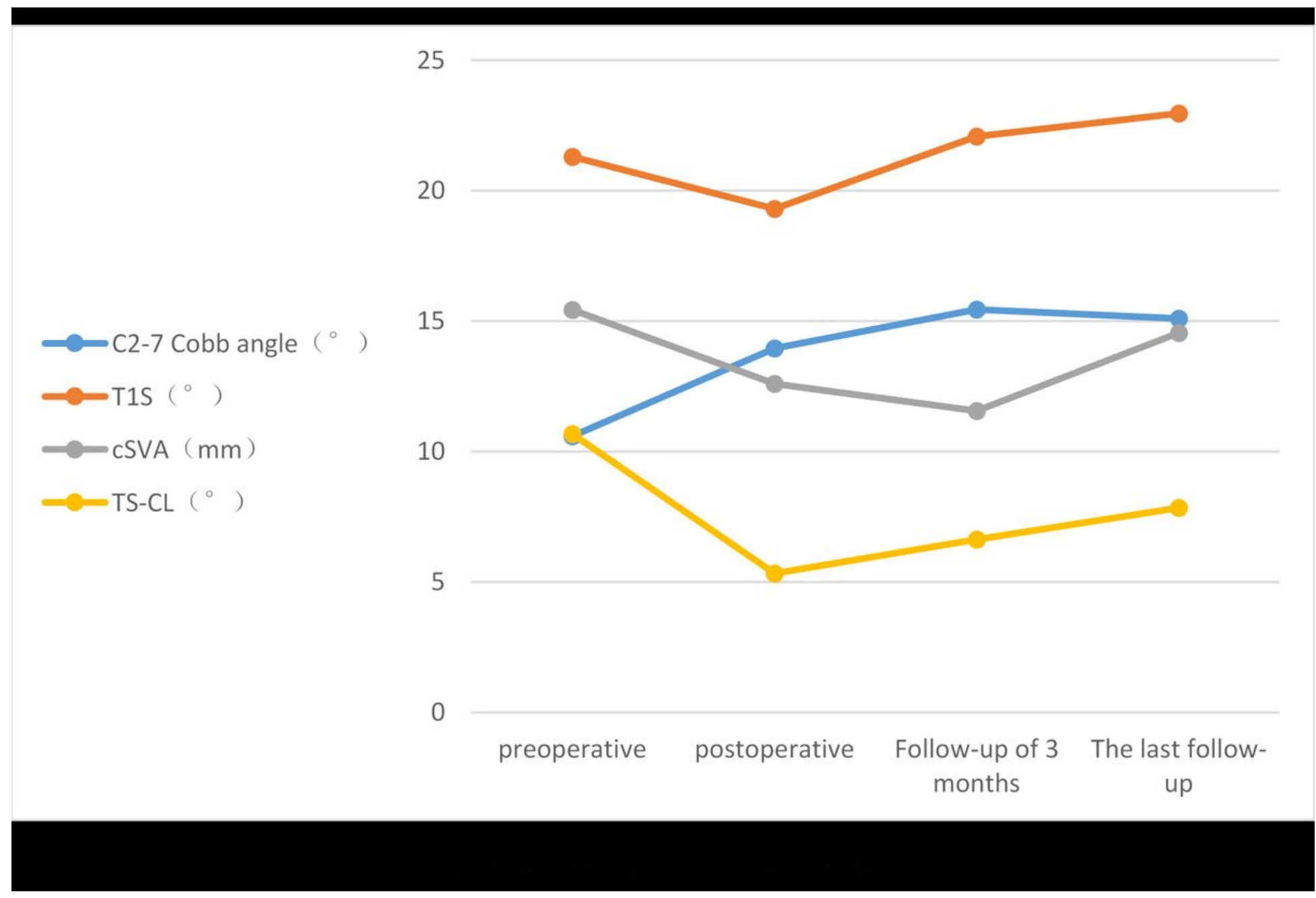

\section{Figure 3}

The variation trend of C2-7 Cobb Angle, T1S, cSVA and TS-CL. 


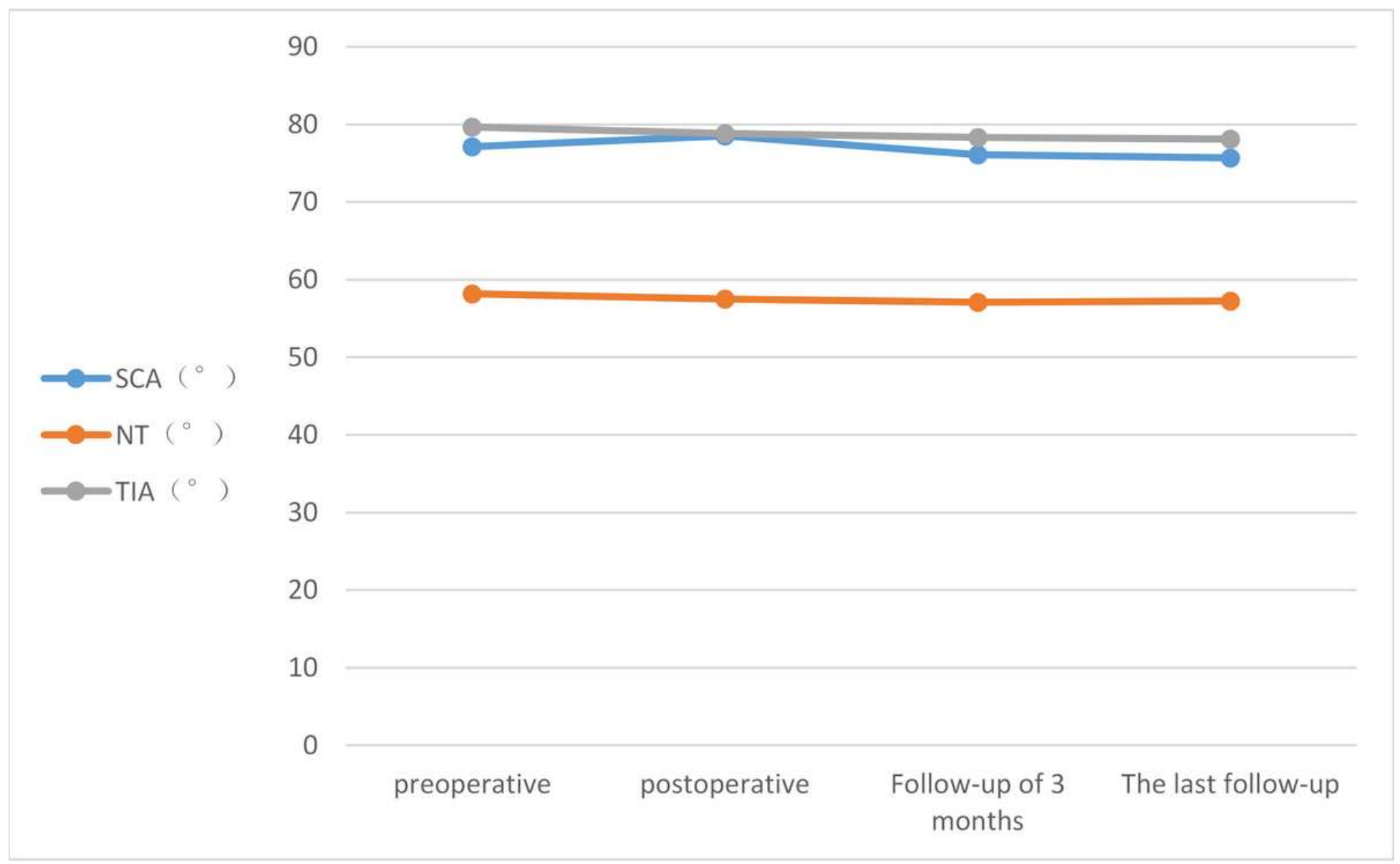

Fig.4 no change in cervical parameters

Figure 4

SCA, NT and TIA do not change significantly 


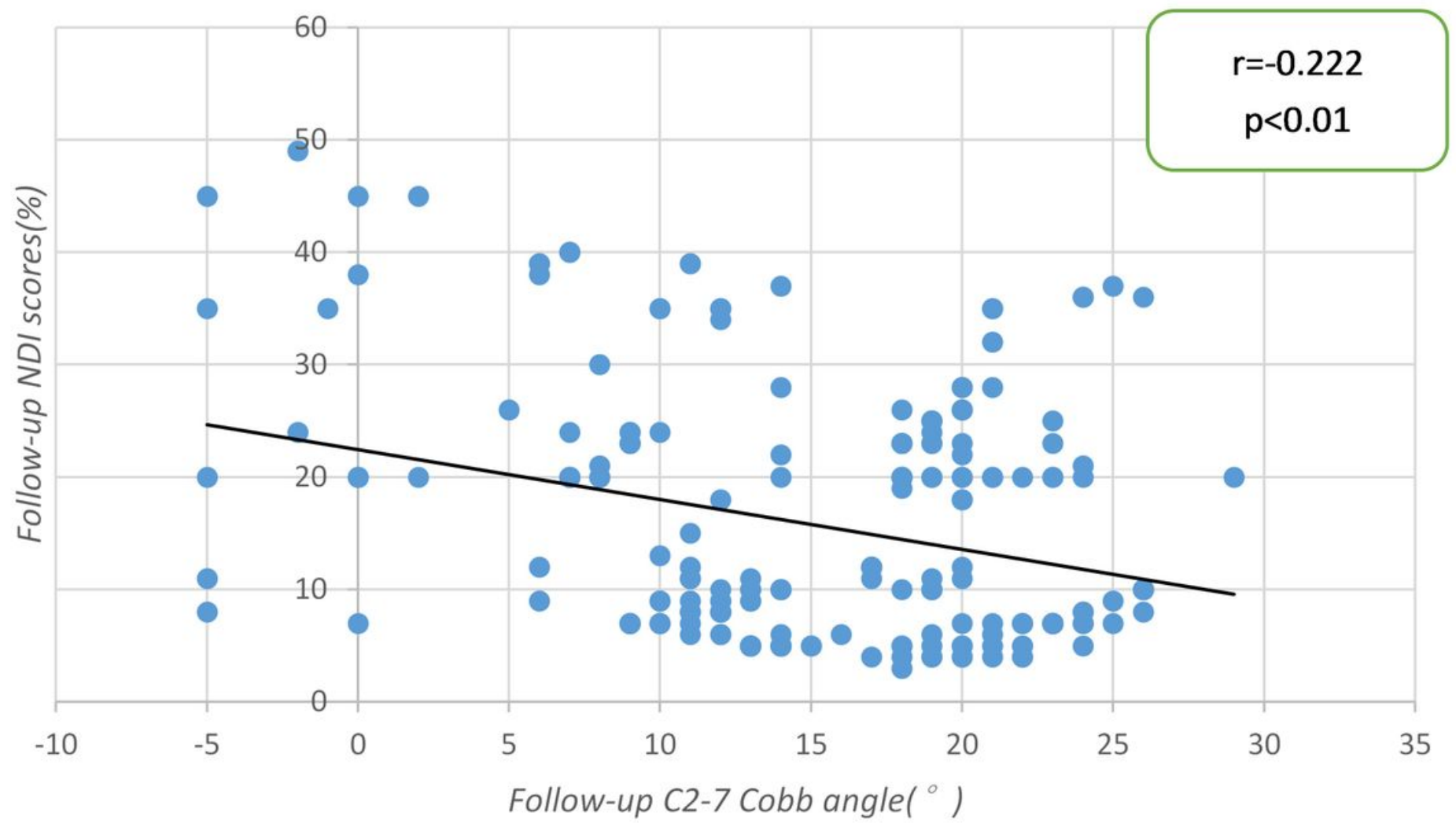

Figure 5

The correlation of follow-up NDI score and cervical lordosis 


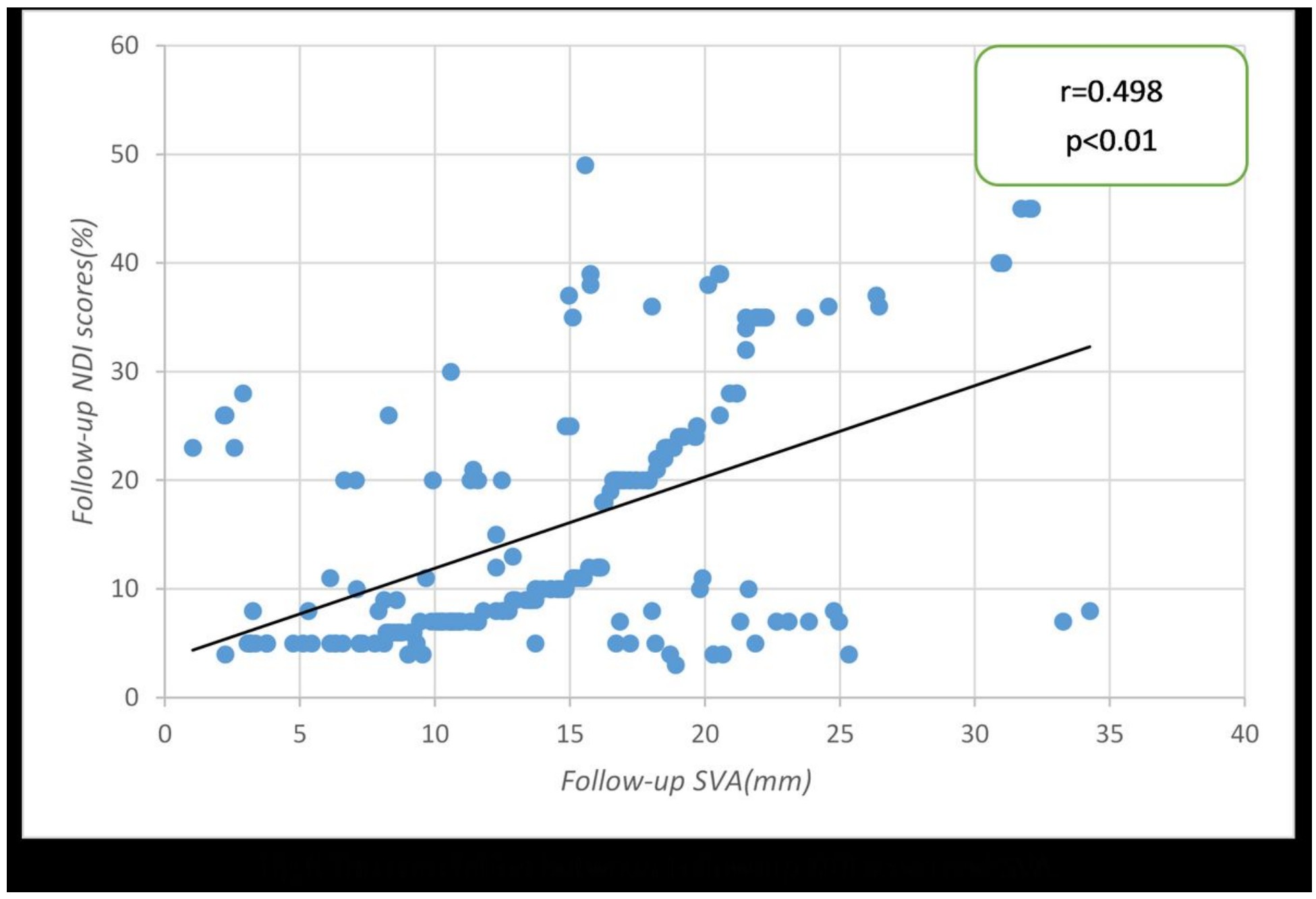

Figure 6

The correlation of follow-up NDI score and SVA 


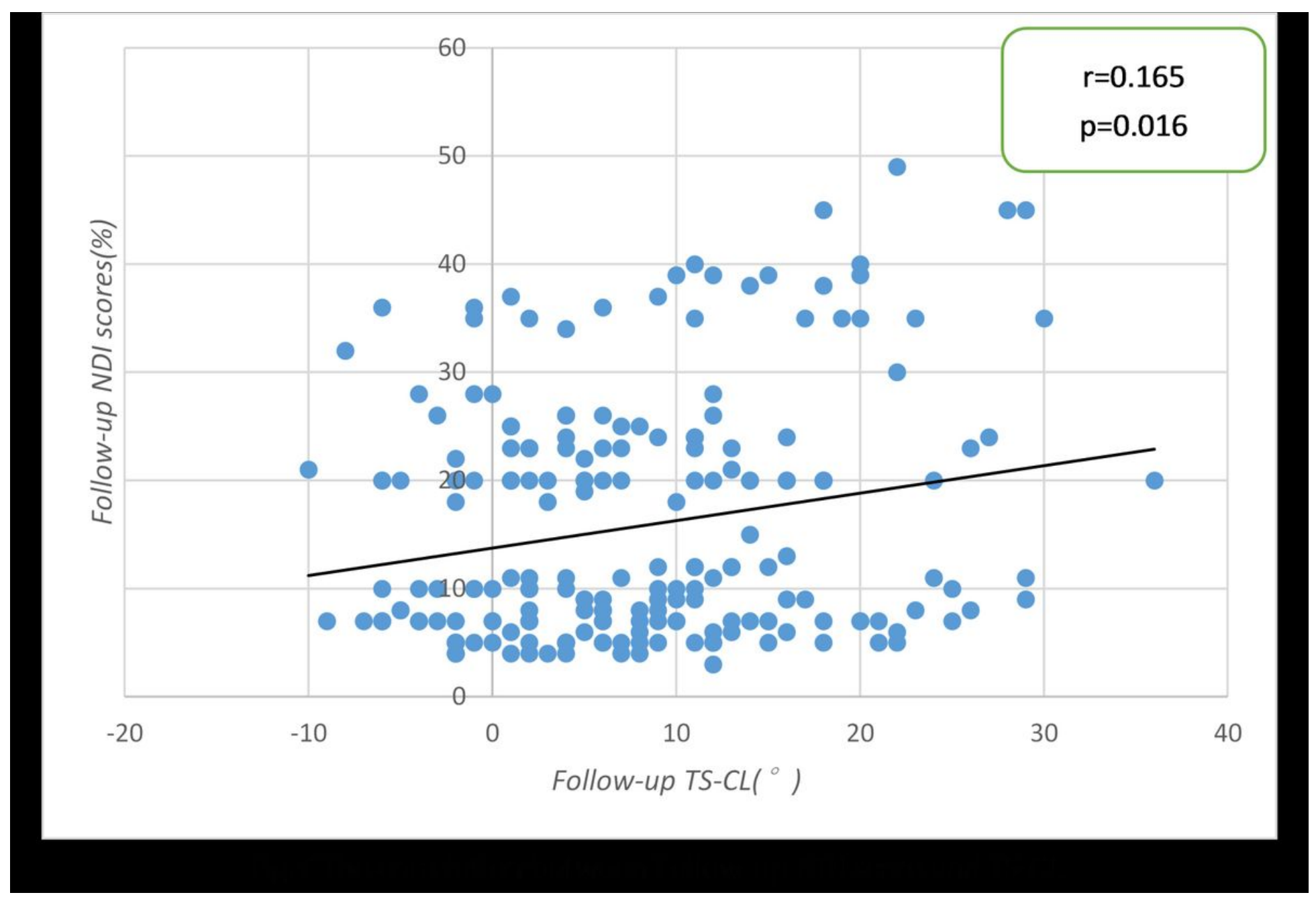

Figure 7

The correlation of follow-up NDI score and TS-CL

\section{Supplementary Files}

This is a list of supplementary files associated with this preprint. Click to download.

- parametrechange.pdf

- NDIchange.pdf 